\title{
Disability Studies
}

Canadian Journal of Disability Studies

Published by the Canadian Disability Studies Association

l'Association canadienne d'études sur le handicap

Hosted by The University of Waterloo

www.cjds.uwaterloo. 


\title{
L'offre culturelle française à l'épreuve de la cécité : étude de cas de l'accessibilité au musée
}

Fréderic Reichhart maître de conférences en sociologie à l'Institut national supérieur de formation et de recherche pour l'éducation des jeunes handicapés et les enseignements adaptés (INSHEA)

frederic.reichhart@inshea.fr

\author{
Aggée Lomo, docteur en histoire, maître de conférences à l'Université de Strasbourg \\ lomo@unistra.fr
}

\section{Résumé}

En France, depuis les trente dernières années, l'accès à l'offre culturelle à destination des personnes malvoyantes et non voyantes connaît un développement important. En s'appuyant sur l'analyse du contenu de portails électroniques, de divers documents institutionnels, d'observations de dispositifs et d'activités, cet article se concentre, dans une perspective sociohistorique, sur l'émergence et le développement de l'accessibilité des espaces et des prestations culturelles; plus précisément, il s'agit d'analyser les éléments constitutifs et structurants de cette offre et d'identifier les différents dispositifs et dispositions mobilisés pour la rendre accessible aux personnes aveugles. En fait, il apparaît que la constitution de cette offre résulte à la fois d'initiatives émanant du mouvement associatif qui milite et s'engage pour le droit à la culture, mais aussi du développement des politiques d'accessibilité de chaque établissement et de celles impulsées par l'État au niveau législatif et institutionnel. Une analyse de l'offre muséale révèle la constitution d'une politique d'accueil en faveur des personnes en situation de handicap notamment de visiteurs non voyants et malvoyants. Elle montre qu'en complément de l'accès au bâtiment (accéder) et dans celui-ci (pénétrer et circuler), l'accessibilité au contenu se développe (utiliser), soutenue par une kyrielle de prestations adaptées.

\begin{abstract}
Over the last thirty years, access to culture for blind and partially blind people in France has improved hugely. Based on analysis of websites and institutional documents and observations of access initiatives and activities, this article concentrates, from a sociohistorical perspective, on the emergence and development of access to cultural spaces and artefacts, more specifically on the analysis of the structures and components of accessibility initiatives which render culture accessible to blind people. We show that improvements in accessibility come, on the one hand, from disability associations who have campaigned for better access and on the other from institution- and state-driven access policies. Our detailed analysis of museum access for blind and partially blind people shows that as well as being able to locate, enter and move around buildings, blind people are increasingly given access to a wide range of cultural services.
\end{abstract}




\section{L'offre culturelle française à l'épreuve de la cécité : étude de cas de l'accessibilité au musée}

Fréderic Reichhart maître de conférences en sociologie à l'Institut national supérieur de formation et de recherche pour l'éducation des jeunes handicapés et les enseignements adaptés (INSHEA)

frederic.reichhart@inshea.fr

Aggée Lomo, docteur en histoire, maître de conférences à l’Université de Strasbourg lomo@unistra.fr

\section{Introduction}

En France, depuis les années 1980, l'accès à l'offre culturelle à destination des personnes en situation de handicap, notamment des personnes malvoyantes et non voyantes ${ }^{1}$, connaît un développement croissant et conséquent : de nombreux conservatoires, écoles de danse, de musique, de chant ou de théâtre, mais aussi des musées proposent des prestations adaptées pour accueillir ce public. En s'appuyant sur l'analyse du cadre législatif, du contenu de portails électroniques, de documents imprimés (brochures, guides, annuaires, etc.), d'observations de dispositifs et d'activités culturelles, cet article analyse l'émergence et le développement de l'accessibilité de l'offre culturelle en faveur des personnes malvoyantes et non voyantes.

Dans un premier temps, il présente la constitution et l'évolution de cette offre dans une perspective sociohistorique à partir de l'analyse des dispositifs institutionnels et des actions et acteurs engagés en faveur de l'accès à la culture. Il souligne que l'accessibilité culturelle s'est construite, d'une part, par les initiatives d'associations de type autosupport ${ }^{2}$, mais aussi du secteur culturel, et d'autre part, de l'intervention de l'État au niveau législatif et institutionnel

\footnotetext{
${ }^{1}$ Les termes « personne non voyante » et «personne malvoyante » ont été retenus à la place du terme «personne aveugle », car ce dernier fait plutôt référence à la non-voyance. En effet, ce travail se réfère à un public confronté à une altération de la fonction visuelle au sens de l'Organisation mondiale de la santé que le terme de personne non voyante et malvoyante exprime bien.

${ }^{2}$ Par autosupport, il faut entendre la mobilisation et l'engagement d'un groupe de personnes ou de pairs concernés par des situations médicales, sanitaires ou fonctionnelles identiques (souvent des personnes toxicomanes, des personnes avec des troubles physiques ou des personnes en situation de handicap), dont le but est de s'entraider et de répondre à des objectifs communs.
} 
avec la création de la Réunion des établissements culturels pour l'accessibilité (RECA), la Commission nationale Culture et Handicap $(\mathrm{CNCH})$ et la signature de multiples chartes.

Une seconde partie traite de l'offre muséale afin d'identifier et d'analyser les conditions opérationnelles facilitant l'accessibilité. Après avoir présenté la constitution progressive de cette offre, ce volet de notre travail met en exergue, dans une perspective plus anthropologique, la dimension globale de l'accessibilité avec la chaîne de déplacement qui combine de manière complémentaire l'accessibilité spatiale garantissant l'accès, la circulation et le repérage au sein de l'espace muséal avec l'accessibilité fonctionnelle permettant l'accès au contenu (Reichhart et Rachedi, 2016).

\section{Accessibilité culturelle et déficience visuelle.}

Le dynamisme associatif au service de l'accessibilité culturelle pour les personnes malvoyantes et non voyantes

Diverses associations se concentrent vers le soutien et le développement de pratiques culturelles, sportives et de loisir à destination de personnes en situation de handicap. Celles concernant spécifiquement la cécité apparaissent au début des années 1970 (Reichhart, 2011), portées par exemple par le Groupement pour une Information Progressiste des Aveugles et Amblyopes (GIPAA). À cette époque, les supports et documents n'étant pas pensés et formalisés en fonction des besoins des personnes malvoyantes et non voyantes, l'information et la culture n'étaient pas accessibles à ces dernières. Parmi les activités proposées par le GIPAA (édition d'une revue, gestion d'une bibliothèque braille et sonore...), certaines prennent des orientations culturelles et touristiques : des week-ends, des conférences, des sorties, dont certaines au théâtre, figurent au programme. Durant les années 1980, une autre association, Handicap Zéro, s'engage en faveur du droit à l'accès à l'information pour les personnes malvoyantes et non voyantes. En plus d'action de sensibilisation, elle propose des services garantissant un accès autonome à l'information générale, la santé, l'emploi, la consommation, la téléphonie, mais aussi aux sports et aux loisirs. Dans ce domaine, par l'intermédiaire de son site internet, elle recense, centralise puis diffuse l'information relative aux loisirs et à la culture dont entre autres les monuments et musées accessibles. De son côté, 1'Association Valentin Haüy (AVH) recense les activités culturelles accessibles qu'elle diffuse dans un agenda consultable en ligne, distinguant les musées, les expositions, les conférences et les concerts. Enfin, la Fédération des Aveugles de France soutient la 
Bibliothèque Numérique Francophone Accessible (BNFA) et organise des évènements culturels dédiés aux personnes présentant des déficientes visuelles.

En complément, des associations proposant des activités culturelles et artistiques comme la danse, la musique, le théâtre accueillent des participants en situation de handicap, dont des personnes malvoyantes et non voyantes. Citons par exemple l'association Musique et Situations de Handicap (MESH) ${ }^{3}$ qui depuis 1984 développe l'activité musicale en faveur des personnes en situation de handicap ; il s'agit d'orienter les parents vers des structures, d'assurer des actions de médiation pour permettre l'accès à l'atelier musical et de proposer des formations aux professionnels (Trémintin, 2012). Dans la même veine, on peut citer l'association CEMAFORRE, créée en 1985, qui a pour but de « développer et de promouvoir l'accès aux loisirs et à la culture pour tous, et tout particulièrement pour les personnes en difficulté pour des raisons de santé ou de handicap (moteur, sensoriel, psychique ou mental) » (CEMAFORRE, s. d.) ; l'Association Européenne pour la Créativité des Personnes Handicapées (EUCREA Europe) fondée en 1987, qui a pour ambition de «favoriser la politique d'égalité des chances pour les artistes handicapés ", met en place et cofinance différents programmes pour promouvoir des actions culturelles par et pour les personnes en situation de handicap (festivals, ateliers, expositions, etc.). Dans le secteur du spectacle, depuis 1990, l'association Accès Culture collabore avec des théâtres et des opéras afin de développer l'accessibilité au spectacle vivant pour les personnes sourdes ou malentendantes ainsi que pour les personnes aveugles ou malvoyantes à l'aide de l'audiodescription. De son côté, Braille et Culture, créée en 1990, « développe et diffuse tout support touristique, culturel et scientifique à l'attention des personnes handicapées visuelles » (Braille \& Culture, s. d.). L'association propose entre autres des actions d'adaptation et de mise en accessibilité de sites en fonction des besoins liés à la déficience visuelle ; les multiples sites adaptés englobent des villes et villages, des monuments et sites touristiques comme des abbayes ou des châteaux, des jardins et sentiers d'interprétation, mais aussi des musées. Ces adaptations et aménagements passent par des outils adaptés comme des textes de visites, des dessins adaptés, des maquettes tactiles, mais aussi des formations à l'accueil des personnes en situation de handicap. Dans le domaine théâtral, l'association Regard'en France Cie, érigée en 1993, joue des pièces classiques dans l'obscurité. Devenue Centre Ressources Théâtre et

\footnotetext{
${ }^{3}$ L'association est également organisme formateur et centre ressource national Musique et Handicap.
} 
Handicap (CRTH) dans les années 2000, elle diversifie ses activités et crée entre autres les «Souffleurs d'Images »; le principe est de mettre en relation des personnes aveugles souhaitant assister à un évènement culturel (comme une pièce de théâtre, un spectacle de danse ou de cirque, une exposition, etc.) avec des étudiants en art, formés au soufflage, qui leur décrivent oralement les éléments visuels du spectacle. Plus tard, la compagnie de danse contemporaine, Acajou, fondée en 2005, intègre des personnes malvoyantes et non voyantes dans ses ateliers chorégraphiques, ses stages de danse et ses sorties culturelles. Enfin, encore dans le domaine musical, l'association de Soutien à l'inclusion de personnes déficientes visuelles dans les lieux d'enseignement de la musique (SIDVEM) «accompagne les enfants et adultes aveugles ou malvoyants dans leur pratique musicale en leur apportant un soutien pédagogique et technique spécialisé » (Association Valentin Haüy, s. d.).

L'intervention de l'État et des collectivités locales

À partir des années 2000, l'État français s'engage enfin en faveur de l'accessibilité culturelle. Dans le cadre du plan gouvernemental en faveur des personnes en situation de handicap, une « instance de dialogue et de consultation entre les ministères chargés de la culture et des personnes handicapées, les principales associations de handicapées, les personnes handicapées elles-mêmes et le milieu culturel et artistique » voit le jour par arrêté en 2001 (Arrêté du 1er février 2001 portant création de la commission nationale « culturehandicap »); il s'agit de la Commission Nationale Culture et Handicap (CNCH) dont le but est de « favoriser l'accès à la culture des personnes handicapées, quelle que soit la nature du handicap, dans le souci que chacun puisse participer pleinement à la vie culturelle »; elle vise entre autres à développer l'accès aux équipements et l'accueil des personnes handicapées, à favoriser les pratiques artistiques amateures et l'accès aux formations et métiers de la culture (Carliez et Fertiez, 2007). Plus tard, en 2003, à la demande du ministre de la Culture et de la Communication, une mission nommée Réunion des établissements Culturels pour l'Accessibilité (RECA) est responsable d'améliorer l'accueil des personnes en situation de handicap dans les établissements culturels ${ }^{4}$. En 2004, parmi les mesures annoncées par la

\footnotetext{
${ }^{4}$ En juin 2018, 28 établissements dont 22 établissements publics relevant du Ministère de la Culture et de la Communication (MCC) et 10 Établissements publics hors tutelle MCC sont signataires de la convention RECA.
} 
RECA, on trouve la création de correspondants handicap dans les services centraux et déconcentrés du Ministère de la Culture et de la Communication.

Ces mesures sont complétées à partir de la fin des années 1990 par une série de conventions et chartes entre différents acteurs et champs. Nous pouvons citer la convention Culture Santé signée en 1999 dont l'objectif est de favoriser le développement des activités culturelles dans les hôpitaux avec entre autres la mise en place de jumelages entre hôpitaux et équipements culturels (musée, théâtre, bibliothèque, conservatoire, etc.) et d'un référent culture au sein des hôpitaux publics ${ }^{5}$. En 2003, une Charte Commune-Handicap est signée entre l'Association des Maires de France (AMF) et les associations porte-parole du Comité d'Entente des Associations représentatives de Personnes Handicapées et de Parents d'Enfants Handicapés. Elle permet aux « communes de manifester, au-delà de leurs obligations réglementaires, leur engagement à donner à la personne handicapée sa place dans la cité, en prenant des mesures concrètes pour répondre aux attentes de ces personnes dans les domaines de la vie quotidienne » et de "promouvoir l'accessibilité de lieux culturels, de loisirs et de tourisme » comme les musées (Charte Commune-Handicap, 2003). Enfin, la convention Culture et Tourisme a pour but entre autres de «promouvoir la politique "culture et handicap" » (Convention cadre Culture - Tourisme, art. 7) et soutient la reconnaissance et valorisation de la marque Tourisme et Handicap développée à partir de 2001 (Reichhart, 2008).

Toutes ces conventions visent à favoriser et renforcer l'accessibilité culturelle en s'appuyant sur le partenariat, la collaboration interinstitutionnelle, interministérielle et une politique déconcentrée et décentralisée. Le travail en réseau se développe et se systématise autour de la coopération des opérateurs et partenaires respectifs qui se décloisonnent et apprennent à se connaître et à collaborer. D'autres chartes se situent sur un versant moins collaboratif, mais plus axé sur la formalisation d'un engagement autour de valeurs, principes, pratiques et règles pour garantir des conditions d'accessibilité ; en ce sens, dans le cadre de la Commission nationale Culture et Handicap $(\mathrm{CNCH})$, une charte d'accueil des personnes handicapées dans les équipements culturels énonce un «ensemble de conseils et de recommandations afin de rendre accessibles et accueillants pour tous les lieux de culture et de pratiques artistiques » (2007). Plus tard, ciblant spécifiquement les téléspectateurs aveugles, la charte de l'audiodescription, signée en 2008 entre le Conseil Supérieur de l'Audiovisuel et la

\footnotetext{
${ }^{5}$ En 2010, une seconde convention s'étend au secteur médicosocial.
} 
mission d'accessibilité des programmes aux personnes handicapées, a pour objectif de constituer « un cadre de référence pour les professionnels, avec des règles très complètes de qualité et de déontologie, des règles nécessaires pour garantir une bonne audiodescription, qui satisfasse, à la fois, les créateurs et les utilisateurs » (Charte de l'audiodescription, 2008).

\section{Vers l'accessibilité de l'offre muséale}

Le musée est un segment emblématique de l'offre culturelle, représenté par près de 10000 établissements répartis sur l'ensemble du territoire, attirant chaque année environ 20 millions de visiteurs du monde entier ${ }^{6}$. Progressivement, cette offre muséale s'est adaptée et ouverte à tous en développant des politiques d'accueil en faveur des personnes n'ayant pas accès à des espaces et activités culturelles et pour lesquelles des dispositifs spécifiques sont mis en place. À titre d'exemple, il peut s'agir de personnes hospitalisées, de personnes âgées, de personnes incarcérées, mais aussi de personnes en situation de handicap. C'est à partir des années 1980 que de plus en plus de musées français développent spécifiquement des actions et dispositifs en faveur de ces dernières. Ainsi, dès 1986, la Cité des sciences et de l'industrie de la Villette met en place une « cellule Handicapés » composée d'une personne sourde et d'une personne aveugle dont le but est de construire des prestations adaptées à chacun (Las Vergnas, 2002). Progressivement, des propositions concrètes et opérationnelles apparaissent et se développent, transformant les musées et leurs prestations (Simonnot, 1993). En novembre 1989, le colloque international intitulé «Des musées ouverts à tous les sens », organisé par l'International Council of Museums (ICOM) et la Fondation de France, illustre le dynamisme engagé et offre une première synthèse et analyse des multiples initiatives mises en place (ICOM, 1991). Toutefois, l'étude de Ribemont et Gilbert montre que l'accueil des visiteurs en situation de handicap est à organiser et à structurer, souvent lié à la demande et programmé le jour de la fermeture du musée (1990). Il faudra attendre les années 2000 pour qu'une véritable politique d'accessibilité se développe ; rythmée par une succession d'enquêtes nationales sur l'accès aux musées et par l'édition de manuels et guides d'accessibilité pour accompagner techniquement et méthodologiquement les établissements, cette politique bénéficie aussi de la loi n 2002-5 du 4 janvier 2002 relative aux musées de France qui affirme les missions des musées de France autour de l'accessibilité et du droit à

\footnotetext{
${ }^{6}$ Le Louvre est considéré comme le «musée le plus visité du monde » avec 7,3 millions de visiteurs en 2016.
} 
l'accès à la culture pour tous ${ }^{7}$. Ce cadre légal précise dans son article 2 que les établissements porteurs de l'appellation «Musée de France» ont pour obligation de «rendre leurs collections accessibles au public le plus large [et] de concevoir et mettre en œuvre des actions d'éducation et de diffusion visant à assurer l'égal accès de tous à la culture ${ }^{8}$ » (Ministère de la Culture, 2002). Cette appellation, assortie de l'utilisation d'un logo, d'une signalétique spécifique et d'une charte graphique, est délivrée par le Ministère de la Culture et impose entre autres aux musées de France d'avoir « en propre ou en réseau avec d'autres musées » un service éducatif notamment chargé des personnes en situation de handicap 9 .

En parallèle, à partir de 2001, la marque Tourisme et Handicap (ATH) identifiant différents équipements et structures accessibles à au moins deux déficiences (Reichhart, 2009) renforce la visibilité des musées accessibles : le cahier des charges concernant les gestionnaires de sites touristiques détaille de manière précise et méthodique l'ensemble des conditions à mettre en place pour concrétiser l'accessibilité au musée en fonction des différents types de handicaps (mental, moteur, auditif et visuel). Concernant le handicap visuel, il évoque par exemple des outils d'aide à la visite (« signalétiques et documents de visites adaptés, œuvres et maquettes manipulables, moulages et plans en relief, cartels et documents de visite en braille, gros caractères et relief, malles pédagogiques multisensorielles, bornes interactives, multimédia... », l'organisation de visites spécifiques («visites et ateliers en langue des signes, visites tactiles, visites thématiques et ludiques, ateliers pédagogiques adaptés... »), des équipements techniques spécifiques («éclairage sans reflets et éblouissements des œuvres et des circulations, signalisation des obstacles, guidage au sol, alarmes lumineuses, main courante et plans inclinés, prêts de fauteuils roulants », les systèmes d'aide à la visite (audioguidage, système infrarouge ou HF, système d'écouteurs individuels), mais aussi la présence des «personnels d'accueil, toutes catégories confondues, sensibilisés ou formés » (Direction Générale des Entreprises, 2005). En 2007, la visibilité et la promotion de l'accessibilité des musées sont dynamisées par l'attribution du prix «Des musées pour tous ». Décerné par la direction des musées de France, il s'agit de valoriser les

\footnotetext{
${ }^{7}$ Parmi ces publications, nous pouvons citer une série éditée par le ministère de la Culture.

${ }^{8}$ Au sens de la loi de 2002, «[1]'Appellation “Musée de France” peut être accordée aux musées appartenant à l'État, à une autre personne morale de droit public ou à une personne morale de droit privé à but non lucratif $»$. Les établissements volontaires se portent candidats et doivent correspondre à un cahier de charges très strict.

${ }^{9}$ Fin mars 2018, 1218 musées publics et privés répartis sur tout le territoire français disposent de l'appellation «Musée de France ».
} 
Reichhart et Lomo, «L'offre culturelle française à l'épreuve de la cécité » CJDS 8.6 (décembre 2019)

aménagements durables en faveur de l'accueil de visiteurs en situation de handicap (Poli, 2010).

\section{L’offre muséale à l'épreuve de la cécité}

Du toucher au multisensoriel

Dès la fin des années 1940, les personnes aveugles interrogent l'accès à l'objet de musée sans la vue (Gilbert, 1999). Mais, c’est véritablement dans les années 1970 qu'une première action significative est à noter. En effet, dans le cadre des ateliers pour enfants, le centre Pompidou organise en 1977 une exposition itinérante intitulée «Les mains regardent ». Destinée aux enfants aveugles de moins de 12 ans afin de les initier au volume et au toucher (Giraudy et Thevenin, 1977), elle promeut une éducation sensorielle pour ouvrir l'enfant aveugle au monde. Cette exposition organisée tel un parcours confirme le toucher en tant que technique de médiation culturelle à développer (Vanbelle, 1987; Griffon et all, 1994). Le toucher transforme les visites et impulse la création d'espaces tactiles (Miguet, 1998). Par la suite, selon l'enquête menée en 1989 par Ribemont et Gilbert (1990) auprès de plus de 1000 musées, en France, 148 musées «s'adaptent aux aveugles » ${ }^{10}$. Sans rédiger une liste exhaustive, nous pouvons citer par exemple, en 1992, l'exposition «L'âge du Silicium » du Muséum national d'histoire naturelle de Paris qui propose des visites pour les personnes malvoyantes via des aides techniques (Gonthier, 1992). En 1995, le Louvre met sur pied sa galerie tactile que nous présenterons dans la partie suivante. En 1996, le Musée d'histoire naturelle de Lille organise des animations durant lesquelles des enfants de 7-8 ans malvoyants peuvent toucher certains animaux du Muséum, visiter l'atelier de taxidermie et bénéficier d'une expérience hors les murs dans une ferme pédagogique (Beckary, 1996). D'autres actions impliquent une approche collaborative avec l'appui de multipartenaires. Dans l'est de la France, depuis 1995, l'association « Au-delà du regard » a pour objectif « la promotion d'actions diverses en faveur du public non voyant auprès des musées, des galeries et des institutions de France » et « la conception, la mise au point et la réalisation de projets concrets, en collaboration avec des musées, galeries et institutions qui acceptent de tels projets. » (L'art au-delà du regard, s. d.). Dans cette optique, elle a co-construit avec le Musée

\footnotetext{
${ }^{10} 124$ musées offrent des facilités d'accès aux personnes handicapées motrices, 76 musées proposent des visites pour les personnes sourdes et 131 musées proposent des ateliers aux personnes handicapées mentales.
} 
zoologique de Strasbourg en 2001 une exposition multisensorielle intitulée « Je touche, tu vois, nous découvrons les animaux », combinant accessibilité tactile, olfactive et sonore, qui a associé des enfants déficients visuels au montage. L'exposition permet de toucher, d'entendre et de sentir une variété de maquettes tactiles et dispositifs sonores et olfactifs (Galico et Laemmel, 2003). On peut aussi noter depuis 2007 l'existence de la mallette pédagogique tactile produite par l'artiste Myriam Colin avec le Fonds Régional d'Art Contemporain (FRAC) Alsace, l'association l'Art au-delà du regard, l'Association des Aveugles et Handicapés Visuels d'Alsace et de Lorraine et le service pédagogique des musées de Strasbourg. Elle est constituée de différents objets, « de supports de différentes natures pour découvrir par le toucher une œuvre de l'artiste Wiebke Siem » (Colin, 2007). Pour Myriam Colin, l'objectif est « de donner accès au monde de l'art aux non-voyants et malvoyants, mais aussi une entrée tactile aux voyants » (Colin, 2007). L'approche tactile, démarche d'accessibilité associée aux visiteurs aveugles, s'ouvre à tous les publics.

L'accessibilité culturelle remet en cause la vue, en tant que sens et fonction sacralisés dans l'expérience muséale. En fait, concernant les personnes aveugles, l'accessibilité culturelle s'est longtemps concentrée et réduite au braille et à la seule lecture tactile des cartels. Progressivement, le braille perd de son prestige et de sa pertinence ; rédiger un texte en braille pour présenter une œuvre nécessite une sélection du contenu : s'il importe que le texte en braille ne soit pas long pour ne pas augmenter la taille du cartel, il faut aussi qu'il ne soit pas trop long à lire, car il le sera par une seule personne à la fois (Chauvey, 2010). Pour pallier cela, des planches individuelles rédigées en braille peuvent être à la disposition des visiteurs. Les progrès technologiques avec d'abord les audioguides puis les applications smartphones permettront de dépasser ces contraintes en individualisant l'accès au contenu. En fait, au-delà de la résistance de certains conservateurs et directeurs, le musée, autrefois, lieu par excellence dans lequel on regarde entame sa mutation pour se transformer en un lieu dans lequel on touche et on écoute. La vue, combinée à d'autres sens, comme le toucher, l'audition et parfois l'odorat aboutit au développement des approches multi sensorielles.

En même temps, de nombreuses ressources sont éditées, composant une riche littérature qui diffuse recommandations et conseils (Grandjean, 1987 ; Direction des musées, 1992 ; Aymond, 1992, Grosbois et al, s.d. ; Fauchard et al, 2000, etc.) pour faciliter l'accueil des visiteurs aveugles. À partir des années 2000, plusieurs colloques, journées d'étude et manifestations scientifiques se consacrent à l'accessibilité culturelle et artistique, incluant les musées. Mais il faudra attendre le colloque intitulé Handicap visuel et exposition, organisé 
par la Cité de la musique en 2011, pour avoir une idée plus précise des « différentes méthodes possibles pour proposer une présentation des collections et des expositions adaptées aux besoins et aux attentes du public déficient visuel »(Capelle-Perceval et Jules, 2011, p. 3).

Une accessibilité globale et complète à l'offre muséale.

À partir d'observations de terrain et de l'analyse de sites internet et ressources imprimées (plaquette de promotions, guide de visiteur, etc.), nous avons analysé les dispositifs d'accueil de visiteurs aveugles auprès d'un corpus de sept musées parisiens ${ }^{11}$. Ce travail montre la prise en compte de la chaîne de déplacement, à travers l'accessibilité spatiale et fonctionnelle. En effet, structurée autour de facilitateurs garantissant le déplacement avec le fait d'atteindre l'établissement à l'aide des transports et de la voirie, d'y pénétrer pour circuler dans le bâtiment ainsi que s'y repérer et s'orienter, l'accessibilité spatiale est complétée par une accessibilité fonctionnelle qui se développe autour de l'utilisation des ressources et prestations et de l'accès au contenu culturel (exposition, œuvres, ateliers, etc.), mais aussi informationnel. Déjà en 1999, Gilbert, chargée de mission auprès des personnes en situation de handicap à la Direction des Musées de France, distingue ces deux types d'accessibilité. Elle explique que «L'accessibilité c'est tout d'abord les dispositions relatives à l'aménagement et à l'architecture des bâtiments [...]. Or, la médiation culturelle joue également un rôle déterminant. Par accessibilité dans ce domaine, on entend produits et services adaptés, informations sur les activités proposées et tarification. » (1999, p. 27).

Concernant l'accessibilité spatiale, plusieurs dispositions et mesures sont mises en place par les établissements. Souvent du personnel est disponible pour recevoir, accueillir, orienter et guider les visiteurs. Parfois, il s'agit d'agents d'accueil de musée ou alors de personnes effectuant un service civique dédiées à cette tâche (Cité de la musique, Musée du Quai Branly...). Sur son site internet, le Musée du Quai Branly met également à disposition un fichier audio à télécharger pour guider le visiteur jusqu'au hall d'accueil. Afin d'appréhender l'architecture et l'agencement général du bâtiment et faciliter par extension le repérage spatial et la circulation du visiteur, certains musées disposent à l'entrée et dans le bâtiment des maquettes en trois dimensions ou alors de plans en relief, occasionnellement complétés par des contenus sonores diffusés via un casque. Ainsi, dès l'entrée de la galerie

\footnotetext{
${ }^{11}$ Musée du Quai Branly Jacques Chirac, Louvre, Cité des sciences et de l'industrie, Philharmonie, Maison Victor Hugo, Cité de l'architecture et du patrimoine, Centre des monuments nationaux.
} 
tactile du Louvre, un plan tactile renseigne la superficie du lieu et la disposition des cuvres. On trouve également des maquettes et plans en relief à l'entrée du Musée du quai Branly et de la Cité des Sciences et de l'Industrie. De plus, de nombreux musées éditent des livrets d'accueil et d'aide à la visite disponibles, en braille ou en gros caractère, pour les visiteurs non voyants et malvoyants.

Pour faciliter la circulation dans les musées, la mise en place de repères podotactiles, comme des bandes de guidage ou d'éveil à la vigilance, sont disposés au sol et des bandes adhésives de signalisation sur les parties vitrées. Par exemple, dans la galerie tactile du Louvre, le visiteur est guidé par une main courante et par une bande podotactile dessinant le parcours à suivre. Quant aux œuvres placées au centre de la galerie, elles sont signalées par des aires podotactiles composées de matériaux et couleurs contrastées. L'éclairage et la luminosité des espaces ainsi que les contrastes des couleurs (entre sol et mur, mur et porte, etc.) sont pensés pour être le mieux adaptés aux besoins des visiteurs malvoyants. Toutefois, n'étant pas possible d'ajuster l'éclairage et le contraste en fonction des particularités de chacun, certains visiteurs peuvent être confrontés à des situations ou expériences inconfortables ou inappropriées posant la question de l'individualisation de l'accessibilité et de ses limites.

Afin de concrétiser l'accès et la découverte du contenu et des activités du musée, plusieurs dispositifs complémentaires existent en fonction de la modalité souhaitée : en effet, les visites autonomes ou « libres » peuvent être complétées par des visites guidées ou encore par la participation à des ateliers et animations culturelles. Le visiteur malvoyant ou non voyant peut choisir de visiter le musée de manière autonome et auto-organisée, seul ou accompagné par un proche ou un ami. À la Maison Victor Hugo de Paris, un livret de visite en gros caractères est à sa disposition et précise les œuvres autorisées à être touchées. En complément, des agents du musée sont disponibles pour indiquer l'emplacement de ces œuvres et faciliter leur découverte par le public. De nombreux musées proposent des audioguides à la fois pour que le visiteur puisse se repérer, mais aussi pour décrire les œuvres, les interpréter et convoquer les imaginaires. Enfin, les cartels et informations écrites présentant les œuvres sont rédigés en gros caractères contrastés et en braille. Toutefois, ces éléments ne semblent pas être suffisants pour garantir une visite en autonomie, la plupart des musées recommandant ou suggérant d'être accompagné.

Dans certains musées, des parcours accessibles ou adaptés sont mis en place; il s'agit d'œuvres d'art choisies, réparties dans les salles d'expositions, que le visiteur est invité à 
découvrir via des audioguides, des livrets tactiles et des médiations sensorielles. Le Musée du Quai Branly propose un parcours composé de sept objets à effectuer à l'aide d'un guide tactile d'exploration des collections et d'un audioguide. Le guide, en version à imprimer en thermogonflé et à imprimer en couleurs, reproduit en relief chaque objet qui est décrit en gros caractères ou en braille ${ }^{12}$. Toutefois, rappelons que l'impression en thermogonflage n'est pas aisée et nécessite l'utilisation d'un équipement spécifique à savoir du papier et un four. Le guide est complété par l'audioguide qui accompagne la lecture tactile de chaque objet du parcours. La Cité de la musique développe un parcours d'exploration des instruments de la collection permanente, privilégiant une approche sonore, visuelle et tactile. Intitulé « Touchez la musique », il est élaboré dans une démarche de mixité et d'accessibilité universelle, à destination de tous les publics. Il s'agit d'un parcours composé de cinq instruments à toucher, présentés individuellement sur une table multifonction disposée aux différents étages du bâtiment. Chacune des cinq tables est conçue de manière identique : à gauche, l'instrument à toucher est fixé en position de jeu, au centre des informations techniques et historiques sur l'instrument sont présentées et commentés à l'aide de photos, gravures et dessins, à droite, un emplacement est dédié à des expériences ludiques pour appréhender de façon sensible le fonctionnement de l'instrument (échantillons de matériaux à toucher, tuyaux et circuits d'air, extraits sonores à reconnaître, etc.). Enfin, à l'extrême droite un écran encastré sur plan incliné diffuse une séquence audiovisuelle, sorte de mode d'emploi visuel invitant à toucher l'instrument, la table et les dispositifs interactifs. Par contre, il est difficile de repérer les cinq tables qui sont placées parmi les instruments des collections permanentes. Quant au Louvre, il propose un parcours «Le corps sans le voir » à partir de huit œuvres d'une exposition, à destination d'un public malvoyant. Les œuvres sont accessibles par l'intermédiaire de huit fichiers audio à télécharger sur le portail du musée. Il convient de souligner la particularité du Louvre, qui a spécifiquement dédié deux espaces aux situations de handicap : la galerie tactile et la petite galerie. Inaugurée en 1995, la galerie tactile présente des expositions thématiques spécifiquement conçues pour les publics en situation de handicap (Miguet, 1998). Dans une vidéo promotionnelle, Maria Pia Vitali, chef de l'unité accessibilité et aide à la visite à la direction de la politique des publics et de l'éducation artistique du Musée du Louvre, explique que la galerie tactile « propose des moulages présentés dans le Musée du Louvre (...) que l'on

\footnotetext{
${ }^{12}$ Le thermogonflage est une technique qui met en relief des tracés simples imprimés sur un papier microcapsule en réaction à la chaleur qui fait gonfler l'encre.
} 
Reichhart et Lomo, «L'offre culturelle française à l'épreuve de la cécité » CJDS 8.6 (décembre 2019)

peut toucher, appréhender avec les mains (...) ils sont à destination première de nos publics handicapés visuels, mais c'est un espace ouvert à tous » (Musée du Louvre, 2014). La sixième exposition intitulée «Sculpter le corps » comporte 18 moulages en plâtre ou en résine, avec des cartels en braille et gros caractères. Les visiteurs sont invités à toucher les moulages : les plus petits sont installés sur des socles tournants et les plus imposants sont exposés de manière à pouvoir en faire le tour. Quant à la Petite galerie, affirmée comme un lieu d'accueil privilégié des visiteurs handicapés, elle peut être visitée à l'aide d'applications à télécharger pour suivre des parcours commentés et des visites audiodécrites. Bien que ces parcours garantissent l'accès au musée et à son contenu, ils mettent en doute la possibilité du visiteur de choisir et d'orienter sa visite, lui imposant des œuvres précises à découvrir. En ce sens, l'accessibilité se pose en tant que condition déterminant le libre choix du visiteur.

La découverte du musée et de son contenu peut aussi s'effectuer de manière « organisée » et encadrée via des visites guidées. Celles à destination des personnes aveugles sont individuelles ou collectives, programmées ou réalisées sur commande et réservation pour des groupes constitués. Structurées autour d'autres sens que la vue, elles sont de nature descriptive insistant sur la composition et structuration des œuvres (tableaux, sculpture, etc.), mais aussi des lieux et de l'aménagement de l'espace comme celles proposées par le Centre des Monuments Nationaux. D'autres visites sont tactiles, c'est-à-dire offrant aux visiteurs la possibilité de toucher des objets, souvent des fac-similés, des maquettes ou encore des dessins en relief. Parfois, des supports thermogonflés sont aussi utilisés pour mettre en relief des peintures comme à la Maison Victor Hugo.

En complément des visites autonomes ou guidées, des ateliers et animations accessibles émergent. Le Musée du quai Branly organise des ateliers de musique et de danse accessibles aux participants aveugles ainsi que des ateliers basés sur une approche sensorielle combinant écoute et toucher. Ainsi, en collaboration avec l'association Percevoir, les « lectures partagées dans le noir » proposent « une découverte sensorielle et originale autour d'un texte lu en braille » et les séances de l'«Aventure d'une œuvre dans le noir » contribuent à découvrir un objet de la collection par l'écoute et le toucher d'un fac-similé. La Cité des sciences et de l'industrie propose diverses animations adaptées à la déficience visuelle; un jeu (la mémoire en question) dispose de supports rédigés en braille et en gros caractères, une animation (l'illusion sonore) prévoit des sonagrammes imprimés sur papier thermogonflé et un atelier (silence on tourne) permet la manipulation de maquettes représentant le système solaire et les planètes. Ces initiatives sont complétées par des jeux vidéo à télécharger ou à 
Reichhart et Lomo, "L'offre culturelle française à l'épreuve de la cécité » CJDS 8.6 (décembre 2019)

jouer en ligne également accessibles. Citons aussi l'édition d'ouvrages adaptés : le service Accessibilité de la Cité des sciences et de l'industrie a édité un ouvrage pour les personnes malvoyantes et non voyantes, avec des images en relief coloré et une double écriture, des caractères agrandis et en braille ; le Musée du Quai Branly propose à destination de personnes aveugles et à l'entourage qui les accompagne, un imagier sensoriel composé de textes en braille, commentaires audio et représentations tactiles de 16 œuvres.

Enfin, le prêt et la mise à disposition de matériel méritent aussi d'être évoqués ; des loupes, des jumelles et des lampes torches sont à la disposition des visiteurs selon leurs besoins. Certains espaces disposent de matériel informatique adapté ; au Musée du Quai Branly, un salon de lecture et la médiathèque proposent une machine à lire et des postes d'informatique équipés d'un lecteur d'écran, d'une synthèse vocale, d'une plage braille et d'un scanneur.

\section{Conclusion}

Cet article montre la constitution progressive de l'offre culturelle à destination des personnes malvoyantes et non voyantes, partant d'actions sporadiques, isolées, expérimentales pour aboutir à des prestations adaptées aux besoins du public. À partir de l'expérience des musées, il souligne la mise en place d'une multitude de facilitateurs qui concrétisent l'accessibilité, désacralisant la vue au profit d'approches tactiles, sonores et de plus en plus multi sensorielles. Le principe de chaîne de déplacement est pris en compte et appliqué ; il garantit la continuité de l'accessibilité autour d'une accessibilité spatiale avec l'accueil et la circulation des visiteurs dans le musée et une accessibilité fonctionnelle avec l'accès au contenu et aux œuvres. Mais cette accessibilité mérite d'être abordée au regard de l'expérience de visite et de l'usage des dispositifs mis en place; certains espaces et œuvres sont mal éclairés, des livrets en braille sont non disponibles, car sous clés. De même, cette accessibilité questionne le libre choix, en termes d'égalité, ainsi que l'autonomie du visiteur aveugle : dans les faits, ce dernier n'a pas accès aux mêmes offres que le visiteur voyant, mais plutôt à une offre spécifique composée de parcours et de visites ou ateliers adaptés qui conditionnent, orientent voire limitent ses choix. De plus, l'accès au musée n'est pas totalement autonome, car il est souvent recommandé de venir avec un proche ou une aide humaine : l'accessibilité, distincte de l'autonomie, devient alors autonome ou accompagnée. C'est à partir de ces éléments qu'il faut repenser le rapport à l'art et à la culture au sein des 
Reichhart et Lomo, «L'offre culturelle française à l'épreuve de la cécité »

CJDS 8.6 (décembre 2019)

sociétés contemporaines et plus spécifiquement l'accès au musée dans la production des œuvres d'art, dans leur mise en scène et les processus de transmission. La diversité des configurations des troubles de la fonction visuelle doit conduire les médiateurs à des approches multiformes de l'exposition muséale qui doit être coproduite avec les bénéficiaires ultimes. Ce qui soulève aussi l'adéquation entre offre et demande tout comme cela interroge plus largement les politiques publiques et le regard de la société sur les déficiences sensorielles. 
Reichhart et Lomo, «L'offre culturelle française à l'épreuve de la cécité » CJDS 8.6 (décembre 2019)

\section{Références}

Association des maires de France et des présidents d'intercommunalité. (2003). Charte Commune-Handicap

Association Valentin Haüy. (s. d.). Le SIDVEM. Repéré le 11 juillet 2019 à http://www.avh.asso.fr/fr/lassociation/partenaires/le-sidvem

Aymond, P. (1992). Se déplacer dans l'espace et s'orienter dans une exposition. Communication présentée au colloque Expositions et handicap visuel. Paris, Museum d'histoire naturelle

Beckary, S. (1996). Une classe culturelle pour de jeunes handicapés visuels. La lettre de l'OCIM, 1996(48)

Blanc, A. (2012). Sociologie du handicap ( $2^{\mathrm{e}}$ éd.), Armand Colin

Braille \& Culture (s. d.). Tourisme accessible. Repéré le 12 mars 2016 à https://www.brailleculture.com/

Capelle-Perceval, B. et Jules, C. (2011, mai). Handicap visuel et exposition. Actes du colloque du Musée de la musique. Paris, Cité de la Musique

Carliez, D et Fertier, A. (2007). Accès des personnes en situation de handicap à la culture en région centre, Cemaforre et Direction Régionale des Affaires Culturelles Centre

CEMAFORRE. (s. d.). Présentation. Repéré le 11 juillet 2019 à http://www.cemaforre.asso.fr/cemaforre presentation.php

Chateigner, F. (2013). Handicap, pratiques culturelles et participation sociale, Suresnes : Éd. de l'INSHEA

Chauvey, V. (2010). Le texte au musée pour les visiteurs non-voyants : comment aborder les choix de contenus et de formes? La Lettre de l'OCIM, 2010(132)

Colin, M. (2007). Outils pédagogiques tactiles. [Billet de blogue]. Repéré le 11 juillet 2019 à https://myriamcolin.blogspot.com/p/objets-et-outilspedagogiques.html?view=snapshot

Creux, G. (2009). Pour une analyse des conduites artistiques des travailleurs sociaux en milieu professionnel (Thèse de doctorat). Université de Franche-Comté

Direction des Musées de France. (1992). Des Musées pour tous, Manuel d'accessibilité physique et sensorielle des musées. Paris : Direction des Musées de France

Direction Générale des Entreprises. (2005). Cahier des charges Gestionnaires de sites touristiques

Fauchard, C. et Bringtown, M. (2000). Musées sans exclusive 2000 : conditions d'accès et offre de médiation à l'intention des personnes ayant un handicap visuel. Paris : Direction des musées de France

Galico, A. et Laemmel, C. (2003). Je touche, tu vois, nous découvrons les animaux: Évaluation d'une exposition multisensorielle pour les enfants voyants et non-voyants. Strasbourg: Musée zoologique de la Ville de Strasbourg

Gilbert, C. (1999). Les Musées ouvrent leurs portes à tous les publics. Espace, 1999(161)

Gilbert, C. et Sanchez, J. (1997). L'accessibilité des musées de France. Musées et collections publiques de France, 1997(214)

Giraudy, D., et Thevenin, M-J. (1977). Les mains regardent (exposition itinérante). Paris : Centre Georges Pompidou

Gonthier, E. (1992). Colloque exposition et handicap visuel. Minéraux et fossiles, 1992(216)

Grandjean, G. (1987). L'accueil des personnes aveugles et malvoyantes dans les musées. Paris : Direction des musées de France, service de l'action culturelle

Griffon, P. et al. (1994). Découverte tactile des Demoiselles d'Avignon de Pablo Picasso dans un CRF pour déficients visuels. Journal d'ergothérapie, 16(4), 181-186 
Grosbois, LP. et al. (s. d.). Guide de visite en relief. La Villette, Paris : Parc de la Villette

ICOM. (s. d.). Des musées ouverts à tous les sens : mieux accueillir les personnes handicapées. Paris : Fondation de France

L'art au-delà du regard. (s. d.). Repéré le 11 juillet 2019 à http://www.artaudeladuregard.org/presentation/objectif.php

Las Vergnas, ML. (1992). Des visites confortables pour tous, cahier des charges d'accessibilité aux personnes handicapées. Paris : Cité des sciences et de l'industrie, Direction des expositions

Miguet, D. (1998). Autour de la sensorialité dans les musées. Publics et Musées, 1998(13), 177-182

Ministère de la Culture. (2002). Loi $n^{\circ}$ 2002-5 du 4 janvier 2002 relative aux musées de France. Repéré à

https://www.legifrance.gouv.fr/affichTexte.do?cidTexte=JORFTEXT000000769536

Ministère de la Culture. (2007). Charte d'accueil des personnes handicapées dans les équipements culturels

Ministère de la Culture et de la Communication et secrétaire d'État à la santé et aux handicapés. (2001). Arrêté du ler février 2001 portant création de la commission nationale «culture-handicap ». Repéré à

https://www.legifrance.gouv.fr/affichTexte.do?cidTexte=JORFTEXT000000220306\& categorieLien=cid.

Ministère de la Culture et de la Communication et Secrétariat d'état chargé du Commerce, de l'Artisanat, des Petites et moyennes entreprises, du Tourisme, des Services et de la Consommation. (2009). Convention cadre Culture - Tourisme

Morisset, L. et Gonant, F. (2008). Charte de l'audiodescription. Repéré sur le site du Conseil supérieur de l'audiovisuel : https://www.csa.fr/Media/Files/EspaceJuridique/Chartes/Charte-de-l-audiodescription.

Musée du Louvre (2014). La galerie tactile du Louvre. [Vidéo]

Poli, M-S. (2010). Présentation du prix «des musées pour tous ». Culture \& Musées, 2010(15), 162-163

Reichhart, F. (2009). Les loisirs touristiques en faveur des personnes déficientes ; états des lieux et perspectives en France. Loisir et Société,31(2), 290-313

Reichhart, F. (2011). Le tourisme adapté : fondements idéologiques et institutionnels. Paris : L'Harmattan

Reichhart F. et Rachedi Z. (2016). L'accessibilité de 1975 à nos jours : vers une ville accessible à tous ? Les cahiers de la LCD, 2016(1), 75-90

Ribemont F. et Gilbert C. (1990). La Direction des Musées de France et les personnes handicapées, bilan et perspectives. Paris : Direction des Musées de France

Simonnot C. (1993, mai). Le musée sort de sa réserve. Actes de la Journée d'information sur l'accueil des personnes handicapées dans les musées de Handicap international, Lyon

Trémintin J. (2012). MESH, une association pour la pratique musicale. LIEN SOCIAL, 2012(1067).

Vanbelle, F. (1987). Une approche tactile de la sculpture pour les non-voyants est-elle possible? Bulletin pédagogique Groupement de professeurs et d'éducateurs aveugles et amblyopes, 1987(108), 7-13 